\title{
Peptococcus niger
}

National Cancer Institute

\section{Source}

National Cancer Institute. Peptococcus niger. NCI Thesaurus. Code C86643.

A species of obligately anaerobic, Gram-positive, cocci shaped bacteria assigned to the phylum Firmicutes. This species is nonmotile, nonhemolytic, produces hydrogen sulfide and a black pigment that disappears upon exposure to air, indole and esculin negative, and does not reduce nitrate, ferment carbohydrates, liquefy gelatin or serum, or utilize lactate, starch or hippurate. P. niger is commensal to the human intestinal tract and has been isolated from the human naval and urine of adult women. 